\title{
Non-exposed endoscopic wall-inversion surgery for a gastrointestinal stromal tumor of the stomach: A case report
}

\author{
PRASIT MAHAWONGKAJIT $^{1}$, AJJANA TECHAGUMPUCH ${ }^{1}$ and WORAPOP SUTHIWARTNARUEPUT ${ }^{2}$
}

Departments of ${ }^{1}$ Surgery and ${ }^{2}$ Pathology, Faculty of Medicine, Thammasat University, Pathumthani 12120, Thailand

Received September 4, 2016; Accepted June 23, 2017

DOI: 10.3892/ol.2017.6787

\begin{abstract}
Complete surgical resections are crucial for permanently curing patients with gastrointestinal stromal tumors (GISTs). Laparoscopic wedge resection is a widely accepted surgical treatment, but identifying the tumor margin from a serosal laparoscopic view is challenging when using this technique. Non-exposed endoscopic wall-inversion surgery (NEWS) for patients with gastric GISTs is a novel, minimally invasive surgical technique that may aid in complete resection of the tumor margin by endoscopy and laparoscopy methods, removing the whole layer of the gastric wall and the entire tumor, with decreased risk of peritoneal contamination or tumor spread to the peritoneum. To the best of our knowledge, the present study reports the first use of NEWS for a patient with small gastric GIST in Thailand. A 61-year old female presented with jaundice and was diagnosed with acute viral hepatitis A. At 4 months, the severity of the symptoms had decreased but the serum transaminase in the liver function tests remained elevated. The computed tomography scans incidentally demonstrated a gastric mass that protruded into the lumen. Endoscopic examination revealed a $2.5 \times 2.0-\mathrm{cm}$ sub-epithelial tumor located in the posterior wall of the upper gastric body. The patient was informed and consented to undergo NEWS. No intraoperative or immediate postoperative complications were detected. The patient was discharged 5 days following the surgery. In a follow-up visit 4 weeks subsequent to the surgery, the patient was healthy and without complications.
\end{abstract}

\section{Introduction}

A gastrointestinal stromal tumor (GIST) was the most common mesenchymal tumor of the gastrointestinal tract in the United States of America in 2007 (1). The stomach is the most

Correspondence to: Dr Prasit Mahawongkajit, Department of Surgery, Faculty of Medicine, Thammasat University, 95 Moo 8 Paholyothin Road, Klongluang, Pathumthani 12120, Thailand E-mail: prasit_md@yahoo.com

Key words: gastrointestinal stromal tumor, submucosal tumor, non-exposed endoscopic wall-inversion surgery, stomach frequent anatomical location of these tumors (2). Complete surgical resection, which may avoid tumor rupture or injury to the pseudocapsule, remains the sole method that may result in a permanent cure for patients with GISTs (3). Laparoscopic wedge resection of a gastric GIST represents a widely accepted surgical treatment (4). However, identifying the incisional margin from a serosal laparoscopic view remains challenging. An intraluminal endoscopic view enables the incisional margin to be more precisely identified. Previous studies have reported a local full-thickness resection technique that uses flexible endoscopy and laparoscopy for gastric tumors (laparoscopic and endoscopic cooperative surgery), and uses more precise cutting, which decreases unnecessary and excessive resection that may deform the stomach $(4,5)$. Combining laparoscopic and endoscopic approaches with non-exposure techniques when treating neoplasia is useful for a full-thickness resection, as it may prevent stomach contents from entering the clean abdominal cavity, and provides a transabdominal retrieval route of tumor; however, this technique is limited, as it is associated with the excessive resection of the mucosa and difficulty in determining the resection line $(4,5)$.

The novel technique discussed in the present study for gastric GISTs, known as non-exposed endoscopic wall-inversion surgery (NEWS), is minimally invasive and may aid in more precisely determining the resection line with decreased risk of peritoneal contamination or the exposure of the GIST to the peritoneal cavity (6-9). To the best of our knowledge, the present study describes the first use of NEWS for a patient with a small gastric GIST in Thailand.

\section{Case report}

A 61-year old female presented with jaundice and fatigue in February 2016 at Phra Nakhon Si Ayutthaya Hospital (Ayutthaya, Thailand). The clinical examination and blood test for antibodies demonstrated that the patient was IgM positive and $\mathrm{IgG}$ negative, and the patient was diagnosed with acute viral Hepatitis A. in Phra Nakhon Si Ayutthaya Hospital (Ayutthaya province, Thailand). The patient was treated with supportive care for 2 weeks without fulminant hepatic failure. At 4 months the severity of the symptoms had decreased, but the liver function tests revealed that the transaminase concentration in the blood remained elevated. The computed tomography (CT) scans demonstrated normal liver parenchyma with multiple liver cysts. The CT scans, obtained as 


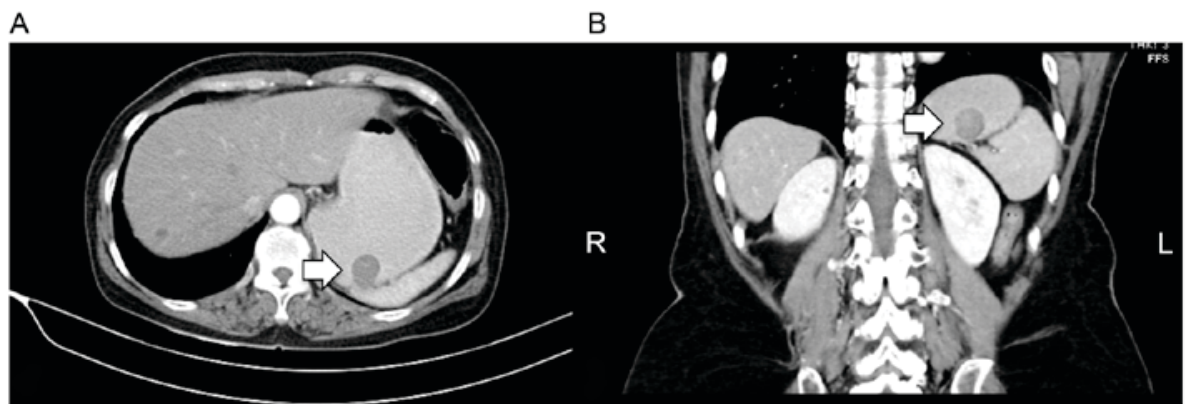

Figure 1. Abdominal computed tomography scanning of a 61-year-old Thai female patient with a small gastric gastrointestinal stromal tumor. (A) Transverse and (B) coronal views of the abdomen of the patient. The tumor (arrows) was located in the posterior wall of the upper gastric body and protruded into the gastric lumen.

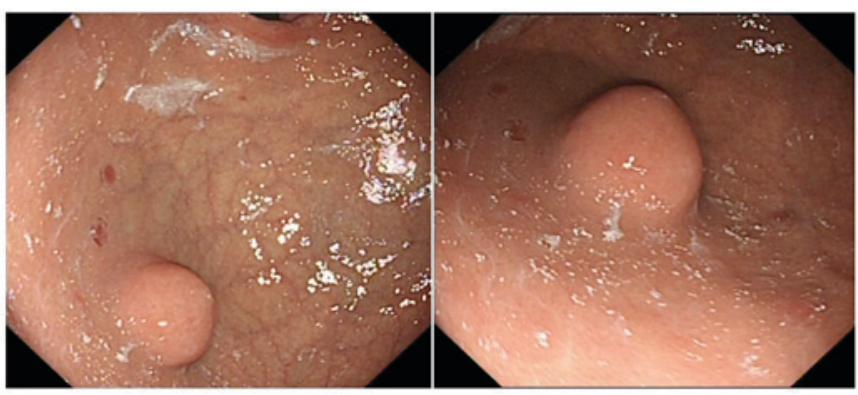

Figure 2. Endoscopic examination of a 61-year old Thai female patient with a small gastric gastrointestinal stromal tumor. Upper gastrointestinal endoscopy revealed a subepithelial lesion in the posterior wall of the upper gastric body.

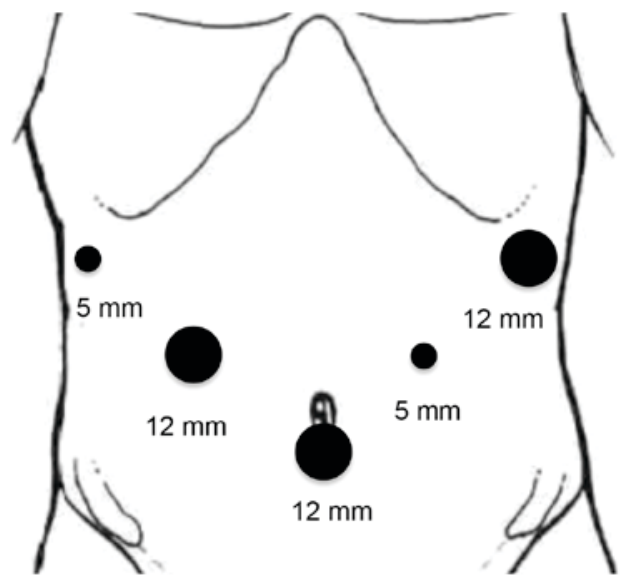

Figure 3. Trocar locations for non-exposed endoscopic wall-inversion surgery. A $12-\mathrm{mm}$ camera port was inserted into the umbilical portion of the abdomen. Pneumoperitoneal gas was also inserted through this port. Subsequently, the 1st trocar was camera port at umbilical portion, and 5- and $12-\mathrm{mm}$ trocars were placed in the left upper and right upper quadrants, two in each quadrant, with five trocars in total.

the patient was assessed for hepatitis, revealed a gastric mass that protruded into the lumen, with no evidence of lymph node or distant metastasis (Fig. 1A and B). The patient was referred to the Department of Surgery, Faculty of Medicine, Thammasat University (Pathumthani, Thailand) where the gastric mass was evaluated. Endoscopic examination revealed a $2.5 \times 2.0-\mathrm{cm}$ subepithelial tumor located in the posterior wall of the upper gastric body (Fig. 2). The patient was informed of multiple treatment options and consented to undergo NEWS. NEWS was performed as follows: The patient was placed under general anesthesia in a supine position with legs apart. A $12-\mathrm{mm}$ camera port was inserted into the umbilical portion of the abdomen as the 1st trocar; pneumoperitoneal gas was also inserted through this port. Subsequently, 5- and 12-mm trocars were placed in the left and right upper quadrants, two in each quadrant, with five trocars in total (Fig. 3). The intestinal clip was attached to the jejunum behind the duodenojejunal junction to prevent endoscopic intraluminal air from passing into the small bowel; the presence of endoscopic intraluminal air in the small bowel would render the laparoscopic procedure more difficult. Flexible endoscopy was performed using a flexible overtube (MD-48518; Sumius; Sumitomo Bakelite Co., Ltd., Tokyo, Japan). Subsequently, multiple mucosal markings were generated around the subepithelial mass using a dual knife (KD-650L; Olympus Corporation, Tokyo, Japan) (Fig. 4A). Next, the gastrocolic ligament was dissected using a laparoscopic LigaSure ${ }^{\mathrm{TM}}$ (LF1637; Medtronic, Dublin, Ireland). A 3-0 braided absorbable suture (Polysorb ${ }^{\mathrm{TM}}$; SL-822; Medtronic, Watford, UK) was used to generate greater curvature and lift for improved exposure to the gastric posterior wall (Fig. 4B). Multiple serosal markings were produced using laparoscopy from the outside of the stomach, opposite the inner mucosal markings previously produced using a Surgiwand ${ }^{\mathrm{TM}}$ II 5-mm cautery with a spatula tip (178099; Medtronic), and guided by a dual knife pressed against the gastric wall (Fig. 4C). The injection solution was prepared with $200 \mathrm{ml}$ Glyceol (glycerin $10 \%$, fructose $5 \%, \mathrm{NaCl} 0.9 \%$ ) and $1 \mathrm{ml}$ of indigo carmine dye. The solution was endoscopically injected into the submucosal layer adjacent to the lesion. The circumferential seromuscular incision was made down to the submucosa strata, which had been stained with indigo carmine dye (Fig. 4D-F). The seromuscular incision was continuously sutured using a 3-0 V-Loc ${ }^{\mathrm{TM}}$ (VLOCL0804; Medtronic) (Fig. 4G) to invert the lesion into the gastric lumen, while an approximately lesion-sized sponge was cut and inserted between the serosal layer of the inverted lesion and the continuous serosal suture line (Fig. 4H). Following inversion into the gastric lumen, images of the lesion were captured (Fig. 4I). The lesion was subsequently removed via endoscopic circumferential mucosal incision using a dual knife, a flexible endoscope (GIF-HQ290; Olympus Corporation) (Fig. 4J) and a VIO300D device (Erbe Elektromedizin GmbH, Tübingen, Germany). The sponge 

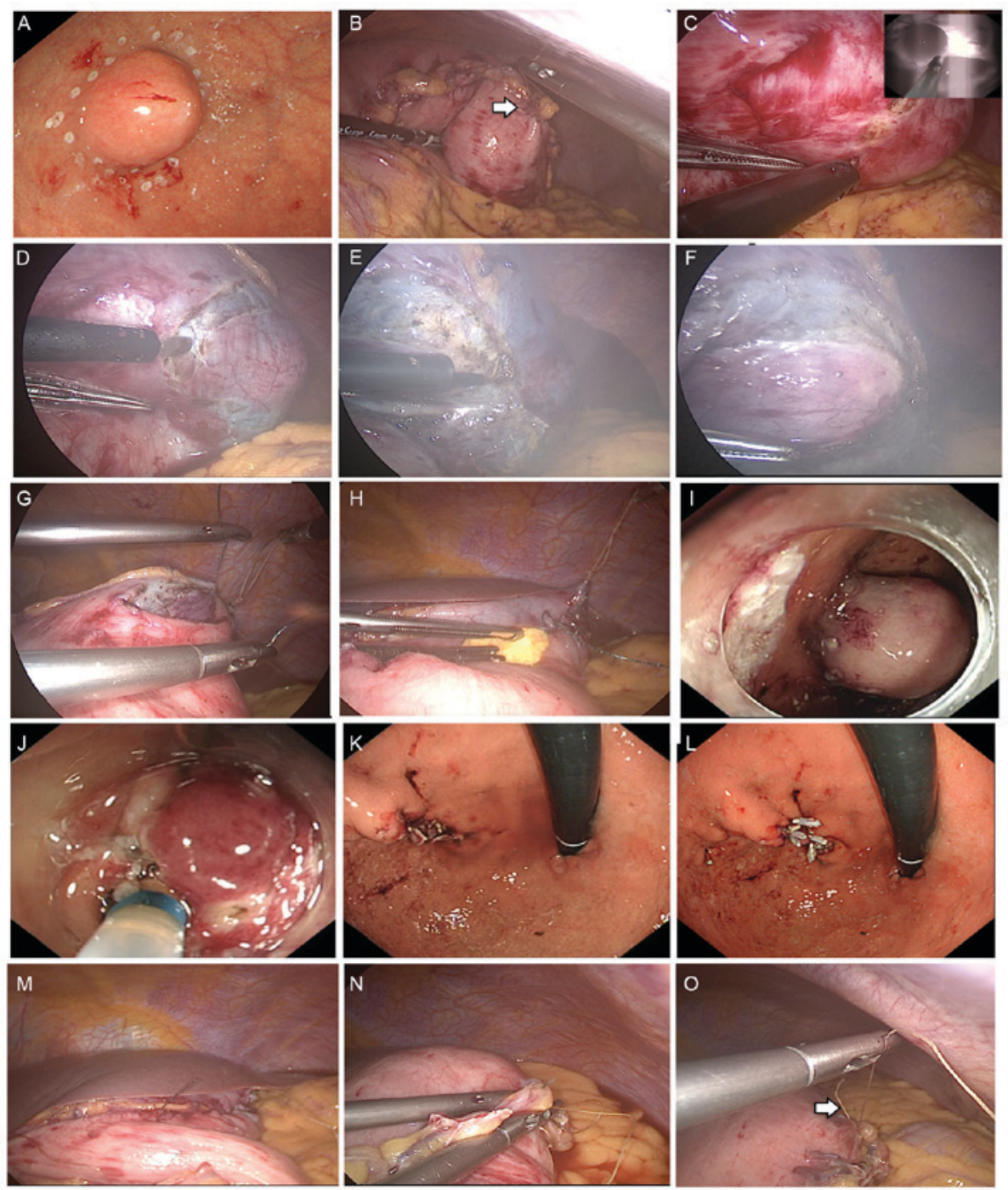

Figure 4. Non-exposed endoscopic wall-inversion surgery procedure. (A) The circumferential mucosal markings were produced using a dual knife. (B) The hanging suture was applied to lift the stomach and expose the posterior wall of the upper gastric body. (C) Laparoscopic serosal markings were guided using a dual knife pressed against the gastric wall. (D) The circumferential serosal incision followed the previous serosal markings. (E) The circumferential muscular incision followed the previous serosal incision. (F) The complete seromuscular incision was performed. (G) The serosal incision was sutured. (H) The sponge was inserted between the serosal layer of the inverted lesion and the continuous serosal suture line. (I) The lesion was inverted into the gastric lumen. (J) The mucosal and submucosal layers were dissected using a dual knife. (K) The dissected suture line following the endoscopic procedure. (L) The mucosal incision was closed using clips. (M) The completed suture, as presented from the laparoscopic view. (N) The pinhole of the hanging suture was subsequently detected by saline pooling on the serosal suture line under endoscopic and laparoscopic views and the suture repaired. (O) The leakage test was performed and checked again.

created traction and prevented the serosal suture from being cut during the sequential endoscopic procedure. The detached lesion and sponge were perorally removed using an endoscopic retrieval device (Roth Net foreign body retriever; 00711052; US Endoscopy, Mentor, OH, USA). The mucosal edges were closed using multiple endoscopic long clips (HX-610-135L; Olympus Corporation) (Fig. 4K and L). An air leakage test was performed through pooling, with normal saline, along the serosal suture line using endoscopic and laparoscopic views (Fig. 4M). The pinhole of the hanging suture was detected and the suture was applied to repair it (Fig. 4N and O). An air leakage test was subsequently performed again. Following the evaluation, the procedure was completed by removing the intestinal clip, port and trocars, and suturing the abdominal incision.

The surgical duration between incision and the closure of the abdominal wall was $219 \mathrm{~min}$. No intraoperative or immediate postoperative complications were detected. The estimated blood loss was $<10 \mathrm{ml}$. The extracted surgical specimen revealed a clear margin, and gastric mucosa and serosa was present on the tumor capsule (Fig. 5A and B). The final pathological report diagnosed the patient with a GIST (maximal dimension, $2.2 \mathrm{~cm}$ ) with a $1 / 50$ high-power field mitotic fig. that was graded low mitotic rate according to the tumor node metastasis histopathological staging (10), which indicated a decreased risk of aggressive behavior (11) (Fig. 5C-F). Immunohistochemical staining confirmed the presence of KIT proto-oncogene receptor tyrosine kinase, the absence of S100 calcium-binding proteins and desmin, and the diagnosis of a GIST (Fig. 5G-I). The staining protocol was as follows: Tissues were fixed in $10 \%$ formalin for at least $8 \mathrm{~h}$ at room temperature prior to IHC staining, and sliced into $3-\mu \mathrm{m}$ thick sections. Hematoxylin and eosin straining 

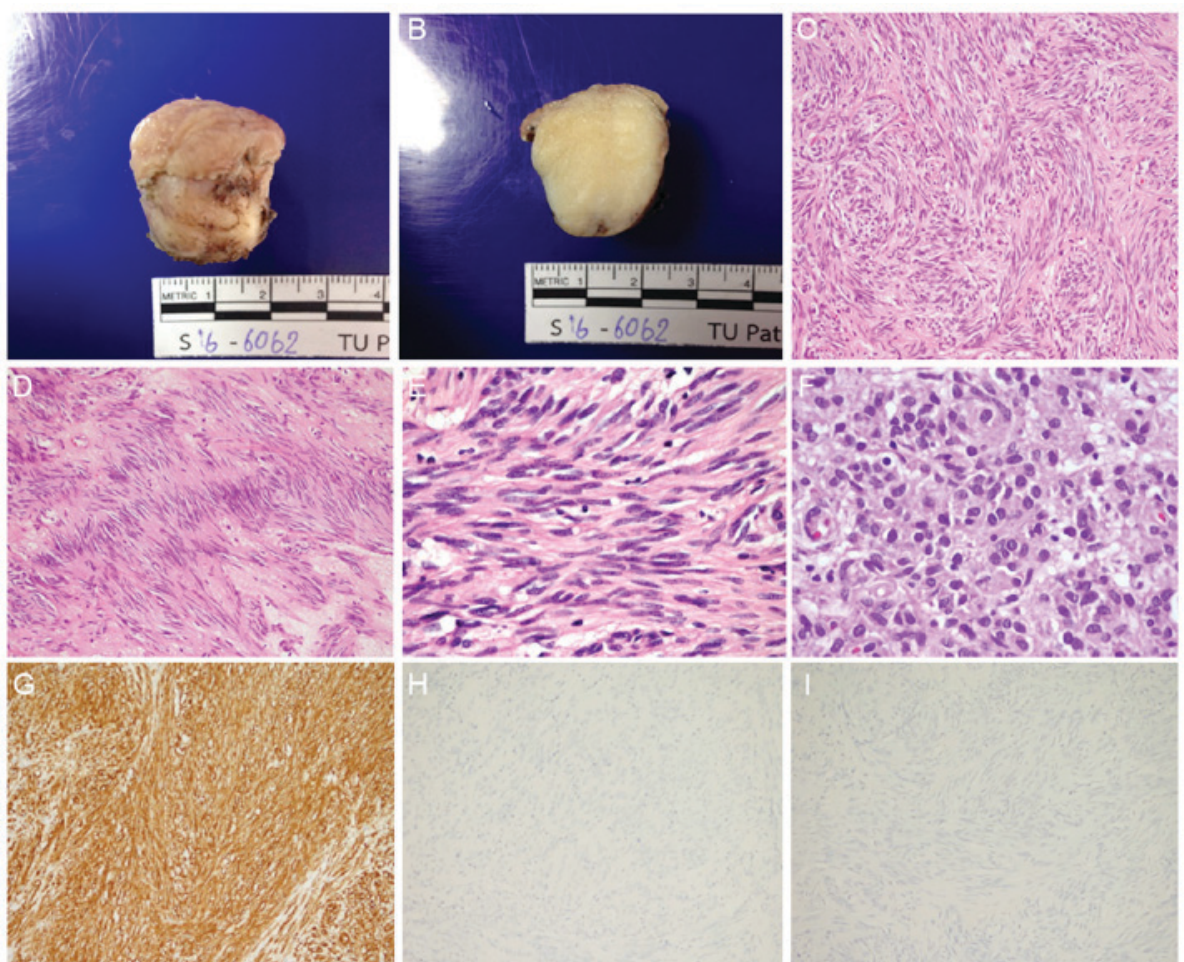

Figure 5. Gross, histopathological and immunohistochemical analysis of the resected specimen. (A) A well-circumscribed, rubbery, white/tan, submucosal gastric nodule with overlying white/tan mucosa. (B) Cut surfaces revealed a homogeneous, fine, fibrillary, white appearance. (C) Hematoxylin and eosin staining showed spindle cell components exhibited intersecting and fascicular pattern (original magnification, x100). (D) Spindle cell components exhibited neurilemmoma-like nuclear palisading (original magnification, x100). (E) Spindle cell components revealed fibrillary cytoplasm, perinuclear vacuolization, bland elongated and wavy nuclei, fine nuclear chromatin, and inconspicuous nucleoli (original magnification, x600). (F) Epithelioid cell components revealed tumor cells with distinct cell borders, eosinophilic cytoplasm, round-to-oval nuclei, variation in nucleus size, fine chromatin and inconspicuous nucleoli (original magnification, x600). (G) KIT proto-oncogene receptor tyrosine kinase demonstrated diffuse immunoreactivity (original magnification, x100). (H) S100 calcium-binding proteins demonstrated a negative result (original magnification, x100). (I) Desmin revealed a negative result (original magnification, x100).

was performed using the Thermo Scientific ${ }^{\mathrm{TM}}$ Gemini AS Automated Slide Stainer (Thermo Fisher Scientific, Inc., Waltham, MA, USA) at $40^{\circ} \mathrm{C}$ for $1 \mathrm{~h} 8 \mathrm{~min}$. Primary antibodies against CD 117 (c-kit, cat no. A4502; concentration, $10.6 \mathrm{~g} / 1$ Dako; Agilent Technologies, Inc., Santa Clara, CA, USA), S100 (cat no. 790-2914; concentration $10 \mu \mathrm{g} / \mathrm{ml}$ ) and Desmin (cat no. 760-2513; concentration $5 \mu \mathrm{g} / \mathrm{ml}$ ) (both from Ventana Medical Systems, Inc., Tuscon, AZ, USA) were incubated with the tissues at $37^{\circ} \mathrm{C}$ for 32,16 and $12 \mathrm{~min}$, respectively. The tissues were incubated with the secondary antibody, an indirect, biotin-free system for detecting mouse $\mathrm{IgG}$, mouse IgM and rabbit primary antibodies (cat no. 760-500; concentration, $<50 \mu \mathrm{g} / \mathrm{ml}$ ) at $37^{\circ} \mathrm{C}$ for $8 \mathrm{~min}$, and visualized with the ultraView Universal DAB Detection kit (Ventana Medical Systems, Inc.) using Benchmark XT IHC/ISH staining module (Ventana Medical Systems, Inc.), and examination with light microscopy at magnifications $\mathrm{x} 10, \mathrm{x} 100, \mathrm{x} 400$ and $\mathrm{x} 600$. The CD117 antibody indicated diffuse and strong cytoplasmic and membranous staining of the spindle cell component, but the sample was negative for S100 and desmin, compatible with GIST (Fig. 5G-I). On the second postoperative day, the condition of the patient was stable and the patient began an oral fluid diet. The patient was discharged 5 days after the surgery. In a follow-up visit 4 weeks following the surgery, the patient was healthy and without complaint regarding oral intake, and the surgical scars had healed without complications. From the surgical and pathological results, the patient exhibited a good prognosis and low risk for metastasis. The requirement for patient consent for publication of this study was waived by the Human Research Ethics Committee of Thammasat University.

\section{Discussion}

Surgical resection with negative margins is a major standard of care for GISTs (3). Multiple previous studies have developed and described endoscopic and laparoscopic approaches for treating patients with gastric tumors that demonstrate advantages of using intraluminal and intraperitoneal procedures during the same surgical period compared with laparoscopic wedge resection $(4,5)$. These procedures increase the precision of the tumor resection margin and minimize unnecessary cutting of the gastric wall. The major risk of these techniques is peritoneal contamination, which may result in infection or tumor cell seeding. NEWS is a novel technique developed by Goto et al (6) that consists of a minimally invasive procedure using laparoscopic surgery and endoscopic intervention with a full-thickness resection of the gastric wall and a decreased contamination risk. NEWS has been used in patients with schwannoma, GISTs, granuloma, an ectopic pancreas and neurinoma $(7,12,13)$, and has been combined with sentinel node basin dissection for early stage gastric cancer $(8,9)$.

The patient in the present study underwent NEWS for the removal of a $<3-\mathrm{cm}$ diameter GIST that could be removed 
perorally. For small subepithelial tumors, an endoscopic ultrasound (EUS) was not performed since EUS may not differentiate GISTs from other hypoechoic lesions from the fourth layer of the stomach and EUS-fine-needle aspiration is associated with a poor diagnostic yield. The NEWS procedure in this patient was successful, resulting in complete removal of the tumor, with full-thickness resection of all layers of the gastric wall without injury to tumor capsule without complications. The pathological report revealed that the gastric GIST and surrounding tumor capsule were completely removed without rupture. The surgical duration (219 $\mathrm{min}$ ) was decreased compared with that of the longest surgery described by Goto et al (13) (range, 171-270 min; mean, $213.5 \mathrm{~min}$ ), and may further decrease in future following repeated performance and increased experience of the technique.

In the present study, the tumor was located in the posterior wall of the upper gastric body, which is more challenging to approach than a tumor located in the anterior wall of the upper gastric body. The present study applied a hanging suture to facilitate approaching the posterior wall. Using a grasper, the hanging suture is held to permit easier access to the tumor, although the traction and movement generated during the procedure may result in an increase in the pinhole size. The pinhole of the hanging suture was detected by inflating the stomach with air using endoscopy and pooling normal saline on the gastric serosa to generate an air bubble in the laparoscopic view. In the present study, an additional suture was applied to repair the pinhole, and an air leakage test was subsequently performed. The suture position was maintained and the results of the air leakage test were observed.

To conclude, the present study described, to the best of our knowledge, the first published cases of minimally invasive NEWS for treating gastric GISTs in Thailand. Further studies are required to confirm that NEWS can be recommended as a standard treatment for patients with small gastric GISTs.

\section{Acknowledgements}

The authors would like to thank Professor Yuko Kitagawa (Chairman), Dr Hiroya Takeuchi and other members of the Department of Surgery (School of Medicine, Keio University, Tokyo, Japan), Professor Naohisa Yahagi, Dr Osamu Goto and other staff of the Division of Research and Development for Minimally Invasive Treatment (Cancer Center, Keio University, Tokyo, Japan) for their technical assistance with the NEWS procedure, and Ryan St. Clair for assistance in editing the English version of the original manuscript.

\section{References}

1. Rubin BP, Henrick MC and Corless CL: Gastrointestinal stromal tumour. Lancet 369: 1731-1741, 2007.

2. Søreide K, Sanvika OA, Søreide JA, Giljacac V, Jureckovad A and Bulusue VR: Global epidemiology of gastrointestinal stromal tumours (GIST): A systematic review of population-based cohort studies. Cancer Epidemiol 40: 39-46, 2016.
3. Nishida T, Blay JY, Hirota S, Kitagawa Y and Kang YK: The standard diagnosis, treatment, and follow-up of gastrointestinal stromal tumors based on guidelines. Gastric Cancer 19: 3-14, 2016.

4. Demetri GD, von Mehren M, Antonescu CR, DeMatteo RP, Ganjoo KN, Maki RG, Pisters PW, Raut CP, Riedel RF, Schuetze S, et al: NCCN task force report: Update on the management of patients with gastrointestinal stromal tumors. J Natl Compr Canc Netw 8 (Suppl 2): S1-S44, 2010.

5. Maehata T, Goto O, Takeuchi H, Kitagawa Y and Yahagi N: Cutting edge of endoscopic full-thickness resection for gastric tumor. World J Gastrointest Endosc 7: 1208-1215, 2015.

6. Goto O, Mitsui T, Fujishiro M, Wada I, Shimizu N, Seto Y and Koike K: New method of endoscopic full-thickness resection: A pilot study of non-exposed endoscopic wall-inversion surgery in an ex vivo porcine model. Gastric Cancer 14: 183-187, 2011.

7. Mitsui T, Nimi K, Yamashita H, Goto O, Aikou S, Hatao F, Wada I, Shimizu N, Fujishiro M, Koike K and Seto Y: Non-exposed endoscopic wall-inversion surgery as a novel partial gastrectomy technique. Gastric Cancer 17: 594-599, 2014.

8. Goto O, Takeuchi H, Kawakubo H, Matsuda S, Kato F, Sasaki M, Fujimoto A, Ochiai Y, Horii J, Uraoka T, et al: Feasibility of non-exposed endoscopic wall-inversion surgery with sentinel node basin dissection as a new surgical method for early gastric cancer: A porcine survival study. Gastric Cancer 18: 440-445, 2015.

9. Goto O, Takeuchi H, Kawakubo H, Sasaki M, Matsuda T, Matsuda S, Kigasawa Y, Kadota Y, Fujimoto A, Ochiai Y, et al: First case of non-exposed endoscopic wall-inversion surgery with sentinel node basin dissection for early gastric cancer. Gastric Cancer 18: 434-439, 2015.

10. Bosman FT, Carneiro F, Hruban RH and Theise ND (eds): WHO Classification of Tumours of the Digestive System. 4th edition. IARC Press, Lyon, France, pp417, 2010.

11. Miettinen M, Fletcher CDM, Kindblom LG and Tsui WMS: Mesenchymal tumours of the stomach. In: WHO Classification of Tumours of the Digestive System. Bosman FT, Carneiro F, Hruban RH and Theise ND (eds). 4th edition. IARC Press, Lyon, France, pp74-75, 2010.

12. Kim DW, Kim JS, Kim BW, Jung JY, Kim GJ and Kim JJ: Non-exposed endoscopic wall-inversion surgery for gastrointestinal stromal tumor of the stomach: First case report in Korea. Clin Endosc 49: 475-478, 2016.

13. Goto O, Takeuchi H, Sasaki M, Kawakubo H, Akimoto T, Fujimoto A, Ochiai Y, Maehata T, Nishizawa T, Kitagawa Y and Yahagi N: Laparoscopy-assisted endoscopic full-thickness resection of gastric subepithelial tumors using a nonexposure technique. Endoscopy 48: 1010-1015, 2016. 